\title{
Broadband optical measurement of AC magnetic susceptibility of magnetite nanoparticles.
}

\author{
R. Soucaille, ${ }^{1, \text { a) }}$ M.E. Sharifabad, ${ }^{2}$ N. D. Telling, ${ }^{2}$ and R. J. Hicken ${ }^{1}$ \\ ${ }^{1)}$ Department of Physics and Astronomy, University of Exeter, Stocker Road, Exeter EX4 4QL, United Kingdom \\ ${ }^{2)}$ School of Pharmacy and Bioengineering, Guy Hilton Research Centre, University of Keele, Thornburrow Drive, Hartshill, \\ Stoke-on-Trent ST4 7QB, United Kingdom \\ (Dated: January 23, 2020)
}

Characterization of magnetic nanoparticles in solution is challenging due to the interplay between magnetic relaxation and agglomeration. The AC magnetic susceptibility of magnetite nanoparticles in water has been studied using magneto-optical methods in the frequency range $10 \mathrm{~Hz}$ to $250 \mathrm{kHz}$. The Faraday effect is detected simultaneously with changes in the fluid configuration. It is shown that the relative sensitivity to the magnetic and structural response can be adjusted by varying the wavelength, paving the way towards spatially resolved studies at the micro-scale.

Magnetic hyperthermia exploits heating from the hysteresis cycle of magnetic nanoparticles (MNP) to locally increase the temperature of malignant cells above a critical value of $43-45^{\circ} \mathrm{C}$ and induce their death ${ }^{1}$. The choice of MNP is limited by the need for sufficiently large magnetic moment while keeping low toxicity within the human body, so that magnetite MNPs are commonly used ${ }^{2,3}$. However, careful characterization of the MNP dynamics stimulated by an AC magnetic field is necessary, since the choice of field amplitude and frequency is limited by the generation of electric currents within the body ${ }^{4}$ that can result in untargeted temperature increases.

The magnetization induced by an AC field may be measured directly using compensated pickup coils. This gives a quantitative measure of the AC susceptibility (ACS) but is difficult to scale to small sample volumes. On the other hand magneto-optical measurements that exploit the Faraday effect can be used to probe the $\mathrm{ACS}^{5-8}$ and can be realised within an optical microscope. The Faraday effect describes the rotation of polarization that occurs when linearly polarized light propagates through a magnetized material. The rotation is proportional to the component of magnetization parallel to the direction of propagation. However the rotation is typically small at $10^{-2} \mathrm{deg} / \mathrm{mm}$. Measurements within a magnetic fluid can be difficult because the field may also affect the dispersion of the MNPs within the solution. MNPs will agglomerate due to dipolar interactions ${ }^{9-13}$, affecting the propagation and scattering of the light within the fluid ${ }^{9-11}$. The Faraday rotation also depends upon the wavelength of the light and is sensitive to the size of the MNPs ${ }^{14,15}$.

In this article a magneto-optical method for the characterization of MNP dynamics is demonstrated. Changes in the spatial configuration of the MNPs (the fluid configuration) are found to influence the measurement, but magnetic and structural effects may be disentangled by making measurements at a number of different wavelengths.

Faraday rotation measurements were made with a tuneable light source consisting of a Xenon lamp (Jobin-Yvon FL1039) and a monochromator (Jobin-Yvon, iHR320) as shown in fig. 1. To maximise the signal to noise ratio, the entrance

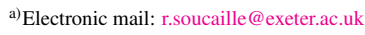

and exit slits of the monochromator were fully opened, resulting in a bandwidth of $50 \mathrm{~nm}$. The emitted light was then passed through a calcite Glan-laser polarizer. After passing through the sample, the beam was split into two components with orthogonal linear polarization using a Thompson prism. The intensities of the two beams were recorded using two fiber optic coupled photodiodes that formed part of a balanced bridge detector (Thorlab PCB450A) of $5 \mathrm{MHz}$ bandwidth.

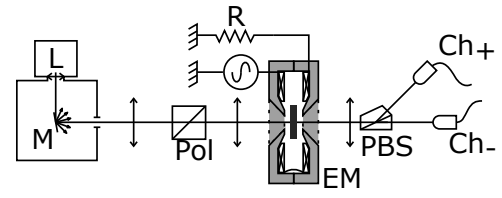

Figure 1. Sketch of the experimental apparatus, with white light source (L) coupled to monochromator $(\mathrm{M})$. The beam is focused with the help of several lenses (double ended arrows) and passed through a polarizer (Pol). The location of the sample cuvette is indicated by the central black rectangle. The electromagnet (EM) generates a magnetic field parallel to the beam direction, and a Thompson beam splitter (PBS) splits the beam into two beams with mutually orthogonal linear polarization $\left(\mathrm{Ch}_{+}\right.$and $\left.\mathrm{Ch}_{-}\right)$. The coil of the EM is connected to an AC power amplifier and a series resistor (R) that allows the current to be measured, while the ferrite yoke is shaded grey.

DC measurements were made using an electromagnet with a steel yoke, and pole pieces with holes drilled through their centre for optical access, which could generate a magnetic field of up to $300 \mathrm{mT}$. The sample was contained within a cuvette made of optical glass (hellma-analytics 110-os-2) that resulted in a path length of $2 \mathrm{~mm}$ within the sample and 2.5 mm within the walls of the cuvette.

The retrieval of the Faraday rotation from the difference output of the bridge detector $\left(\mathrm{Ch}_{+}-\mathrm{Ch}_{-}\right)$requires care because the MNPs react to an applied magnetic field on a timescale comparable to or longer than the duration of the measurement. Specifically, agglomeration of MNPs can modify the optical transmission in a complex manner depending on the wavelength and field history on time scales ranging from miliseconds to hours ${ }^{9}$. The following procedure was used to ensure consistent calibration of the Faraday rotation for all field values within a hysteresis loop measurement. The 
first field value was set and the sample allowed to relax for $250 \mathrm{~ms}$ before the detector output was recorded for different wavelength values. The next field value was then set and the process repeated. Once measurements had been made for all field values, the angle of the polarizer within the incoming beam was set to a new value, and a new set of measurements were made for the set of field and wavelength values chosen previously. Repeating this process, data sets were acquired for polarizer angles through a range of about 3 degrees. Finally, the dependence of output voltage upon polarizer angle was fitted to a straight line for each pair of field and wavelength values. The Faraday rotation angle was taken to be the polarizer angle required to null the output, which was then plotted versus field for each wavelength value. A vertical offset was removed so that zero optical rotation was obtained for zero applied magnetic field. The offset contains a first component due to the initial setting of the input polarizer being arbitrary, and a second that is wavelength dependent and due to the use of non-optimal polarizing optics. An example of the resulting hysteresis loop is shown within the inset of fig. 2 (b).

High frequency measurements were made with an electromagnet based upon a standard ferrite transformer core material (ER64/13/51-3F36, Ferroxcube) with cut-off frequency of $2 \mathrm{MHz}$, to reduce eddy current losses. The core was ground to the desired dimensions, with optical access through the pole pieces, while a $5 \mathrm{~mm}$ air gap allowed a cuvette to be placed between the pole pieces. The driving coil was split around the two pole pieces and was composed of 36 loops of Litz wire ( 800 strands, $40 \mu \mathrm{m}$ diameter per strand). A maximum induction of $50 \mathrm{mT}$ could be obtained for a current of $7 \mathrm{~A}$. For frequencies higher than $3.6 \mathrm{kHz}$, the power amplifier (LPA05B from N4L) cannot provide enough current to reach the maximum field, and the product of magnetic induction $B$ and frequency $f$ is limited to $B f<180 \mathrm{mT} \mathrm{kHz}$. However this limit may be extended by creating a resonant LC circuit in which a capacitor is placed in series with the electromagnet.

The current delivered by the amplifier is measured via a series resistor $(0.1 \Omega)$ while the field is measured by a pick-up coil $(1 \mathrm{~cm}$ diameter, 4 turn) inserted between the pole pieces. Field calibration is performed through the whole frequency range, at low frequency using a Hall probe, and at higher frequencies using a slab of Tellurite glass for which the Faraday rotation is recorded. The pick-up coil gives an accurate field measurement, but the signal is too small to be measured reliably at small fields and frequencies, and then the field must be inferred from the measured current instead.

For optical ACS measurements, it is necessary to compensate for the frequency dependent impedance of the coil and its leads so that a constant field amplitude is maintained for all frequencies. The system is modeled as an electric circuit, with inductance $L$ in series with resistance $R$, for which the resulting magnetic field is proportional to the current. The value of $L$ is determined by measuring the resonant frequency $f=2 \pi / \sqrt{L C}$ when a capacitance $C$ is connected in series with the coil. The control voltage sent to the power amplifier is required to have the form

$$
U_{\text {com }}=\frac{B}{10 K} \sqrt{R^{2}+(2 \pi L f)^{2}}
$$

where $B$ is the magnetic induction generated by the magnet, $K$ is the conversion factor from current to field (in $T / A$ ), and $R$ includes the resistance of the leads, coil, and series resistor. The factor of 10 accounts for the gain of the power amplifier. The AC field calibration is then used to determine the frequency dependent phase of the AC field. ACS measurements were made by sweeping the frequency of the AC field from high to low values and recording the detector output with a lock-in amplifier. Visual inspection of the cuvette was carried out after each measurement to check for sedimentation of the MNPs.

The transmitted light intensity was also measured separately, after removing all polarizers from the setup, with a single fast photodiode of $10 \mathrm{MHz}$ bandwidth to avoid polarization effects that might occur in the bridge detector. The 2nd harmonic intensity signal is of particular interest and was recorded using a lock-in amplifier. The same field calibration was used as for the ACS measurements, but the phase of the 2nd harmonic intensity signal relative to the command voltage is twice that of the first harmonic ACS signal.

It is first necessary to characterize the wavelength dependence of the Faraday rotation obtained from the solution. The magnetization of superparamagnetic nanoparticles in suspension, and hence the Faraday rotation, may be described by a Langevin curve. The glass of the cuvette and the liquid containing the MNPs, also yield a small optical rotation that is proportional to the applied magnetic field. The total measured polarization rotation may therefore be fitted to a sloped Langevin curve of form

$$
\theta_{F}=\theta_{\text {mag }}\left(\operatorname{coth} \frac{\mu B}{k_{b} T}-\frac{k_{b} T}{\mu B}\right)-v_{\text {lin }} B .
$$

where $\theta_{F}$ is the total Faraday rotation, $\theta_{\text {mag }}$ is the Faraday rotation generated by the MNPs at magnetic saturation, and $v_{l i n} B$ is the additional background that is linear in the magnetic induction $B$.

A single value of $B_{0}=k_{b} T / \mu$ was used to fit equ. 2 to the measured $\theta_{F}$ for all wavelengths. Expanding the coth function in equ. 2 to first order, $\theta_{F}$ is found to be linear in $B$ at small field, with gradient $v=v_{\text {mag }}+v_{\text {lin }}$ where $v_{\text {mag }}=\theta_{\text {mag }} /\left(3 B_{0}\right)$. Fig. 2 shows the wavelength dependence of $v_{m a g}$ and $v_{l i n}$ for solutions of magnetite nanoparticles dispersed in cyclohexane with different dilutions. The results are consistent with previous studies ${ }^{14,15}$ since $\theta_{F}$ associated with the MNPs $\left(v_{\text {mag }}\right)$ changes sign at a wavelength close to $560 \mathrm{~nm}$ and becomes opposite in sign to the linear background $\left(v_{\text {lin }}\right)$ for wavelengths between $400 \mathrm{~nm}$ and $560 \mathrm{~nm}$. For the shortest wavelengths the linear background, $v_{l i n}$, is also affected by the nanoparticle concentration, tending to decrease as the nanoparticle concentration is increased. In contrast, the value of $v_{\text {mag }}$ increases towards larger positive values with increasing concentration as reported in previous studies where $\theta_{F}$ depends on the packing of the MNPs ${ }^{14}$.

Measurements of the frequency dependent ACS are typically used to obtain the relaxation time of the nanoparticle magnetization within the framework of linear response theory. The relaxation rate is identified as the frequency at which a maximum occurs in the imaginary part of the magnetic 

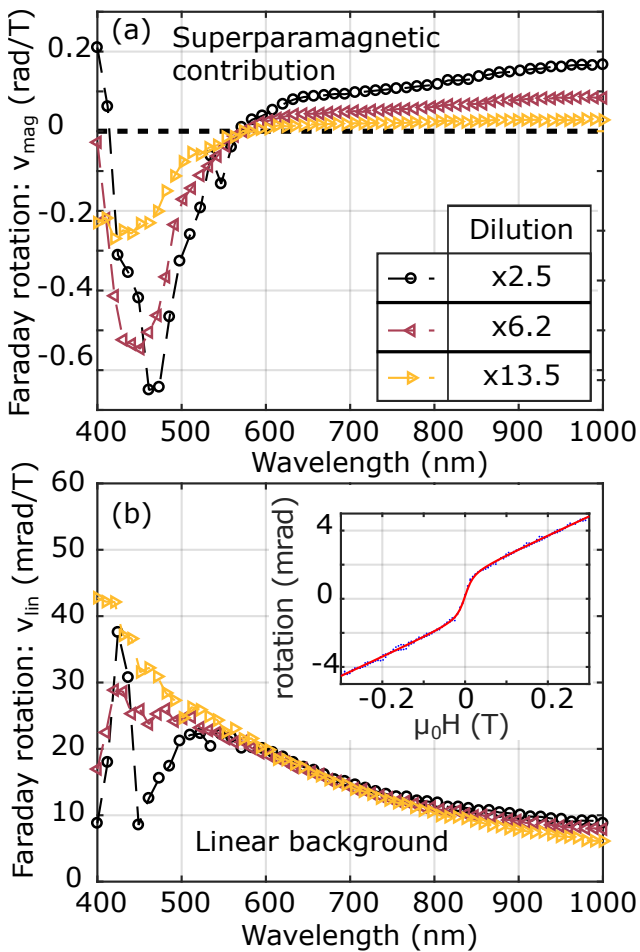

Figure 2. Faraday rotation obtained from solutions of magnetite nanoparticles dispersed in cyclohexane for different dilutions the optical path of the cuvette is $2 \mathrm{~mm}$. The main panels show the wavelength dependence of the parameters (a) $v_{m a g}$ and (b) $v_{\text {lin }}$ defined within the main text. The inset in (b) shows the measured rotation after calibration for a solution of x6.2 dilution at $800 \mathrm{~nm}$ wavelength The solid line is a fit to equ. 2 that allows the Faraday rotation from the superparamagnetic MNPs and the linear background to be extracted. Particle sizes are described within fig. S1 of the Supplementary Material.

susceptibility ${ }^{5,7}$. The magnetization of a MNP can relax by one of two possible mechanisms, firstly by rotation of the nanoparticle associated with Brownian motion, with a relaxation time that depends on the hydrodynamic radius of the nanoparticle, and secondly by Néel relaxation which involves rotation of the magnetization and depends upon the magnetic anisotropy ${ }^{1,16,17}$. In a liquid solution, both mechanisms are involved, and the two relaxation times cannot be determined from a single measurement. Instead it may be necessary to make measurements on solutions of different viscosity ${ }^{2}$, or to measure a reference sample in which the MNPs are immobilized. The signal from bridge detector may result from a combination of different processes since its differential output depends upon both the intensity and change in polarization

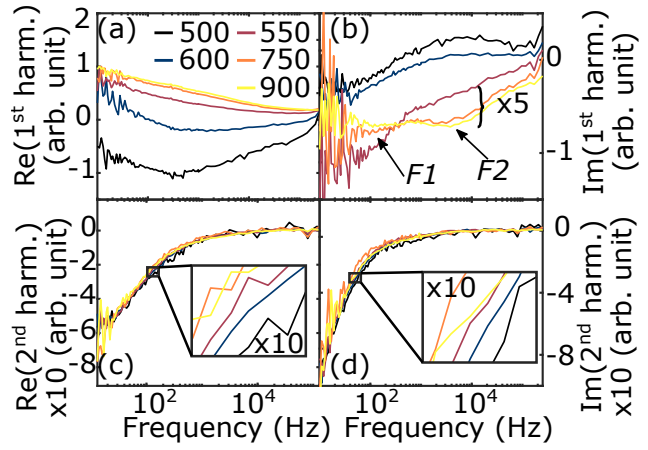

Figure 3. (a) Real and (b) imaginary parts of the difference channel output of the bridge detector recorded during low field ACS measurements. (c) Real and (d) imaginary parts of the second harmonic signal measured with a single photodiode. Results are normalized to the values obtained at $10 \mathrm{~Hz}$, the different wavelength are given in the panel (a) in $\mathrm{nm}$. The imaginary part of 600, 750 and $900 \mathrm{~nm}$ data sets in (b) has been multiplied by a factor 5 . Arrows in (b) indicate two characteristic features of the measurement $F 1$ and $F 2$. The amplitude of the applied AC field was $0.25 \mathrm{mT}$.

state of the light. To determine the magnetic relaxation time, it is necessary to isolate the contribution to the signal from the Faraday rotation of the MNPs.

Low field optical ACS measurements were made on magnetic fluids consisting of magnetite nanoparticles, with size of around $14 \mathrm{~nm}$, dispersed in water. The MNPs were stabilized with an oleic acid coating and the concentration of iron in the fluid was $1.91 \mathrm{mg} / \mathrm{mL}$. Fig. 3 shows the frequency dependence of the optical ACS signal, obtained from the output of the bridge detector, at different wavelengths. Measurements were also performed for different amplitudes of the applied field [fig. 4 and 5]. The optical ACS data in fig. 3 exhibits a change of sign at a wavelength between $550 \mathrm{~nm}$ and $600 \mathrm{~nm}$, suggesting that the Verdet constant of the MNPs may be changing within this wavelength range, as seen previously in fig. 2 . The imaginary part exhibits two features, one at around $100 \mathrm{~Hz}$, (denoted F1), and another one around $10 \mathrm{kHz}$ (denoted F2) indicated by arrows in figs. 3 (b), 4 (b) and 5 (b). These two features have a different dependence on the optical wavelength and the field amplitude. The low frequency feature, $F 1$, has a different characteristic frequency for different field amplitudes. $F 1$ manifests as a minimum, even when $\theta_{F}$ changes sign, while the high frequency feature, $F 2$, instead changes from a minimum to a maximum. Similarly, the relative amplitude of the two features varies with the wavelength as seen in fig. 3 (b), depending on the absorption of the solution and the wavelength dependence of the DC Faraday rotation shown in fig. 2 (a).

The ratio of the part of $\theta_{F}$ arising from the MNPs, to that from the linear background, depends on the wavelength and tends to increase for wavelengths greater than $650 \mathrm{~nm}$ up to the longest wavelength measured. In the high frequency limit, 
the magnetic susceptibility of the MNPs tends to zero, and the optical ACS signal becomes equal to the part of $\theta_{F}$ due to the linear background.

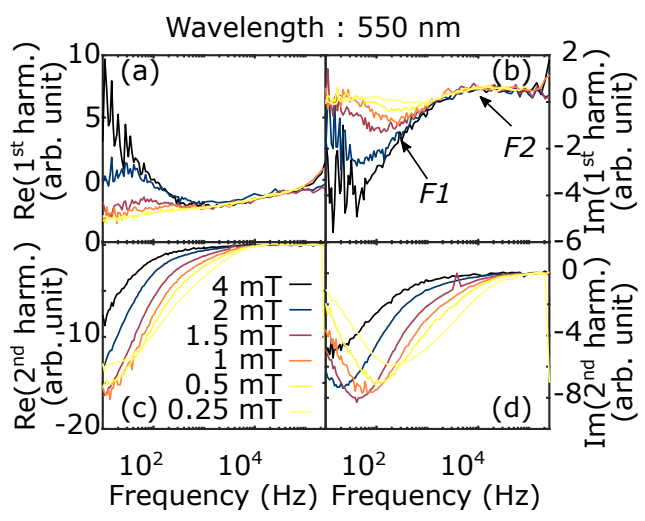

Figure 4. (a) Real and (b) imaginary parts of the optical ACS signal for different magnetic field amplitudes. (c) Real and (d) imaginary parts of the second harmonic component of the transmitted light intensity. All data was recorded at a wavelength of $550 \mathrm{~nm}$. All curves have been normalised to the applied field amplitude.

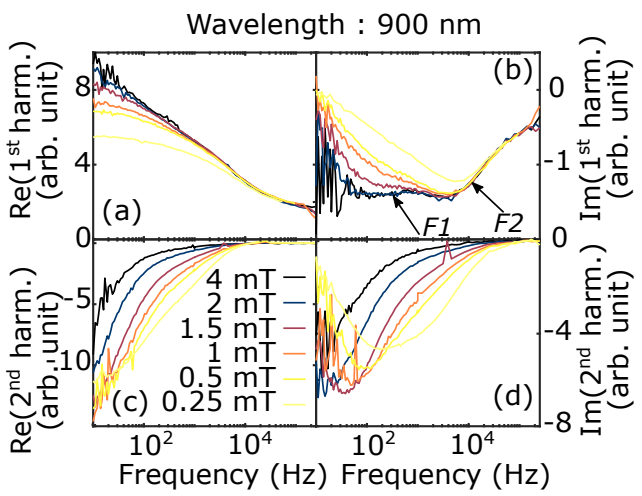

Figure 5. (a) Real and (b) imaginary parts of the optical ACS signal for different magnetic field amplitudes. (c) Real and (d) imaginary parts of the second harmonic component of the transmitted light intensity. All data was recorded at a wavelength of $900 \mathrm{~nm}$. All curves have been normalised to the applied field amplitude.

The feature identified as $F 2$ (fig. 3) is caused by Brownian relaxation of small ( $38 \mathrm{~nm}$ ) clusters of polydisperse nanoparticles, as determined from analysis of conventional inductive AC susceptibility measurements (see Supplementary Material fig. S1). To identify the origin of the feature $F 1$, it is helpful to consider the variation of the transmitted light intensity. Different processes can affect the transmission of the light such as a time dependent aggregation of the nanoparticles that tend to form chain structures ${ }^{9}$, or a reorientation of the easy axes of the nanoparticles or aggregates ${ }^{18}$. Structural change is expected to be an even function of the magnetic field and so should be visible in the second harmonic component of the detector output ${ }^{19,20}$. The measured values shown in figs. 4 (cd) and 5 (c-d) exhibit a single absorption feature in the low frequency part of the spectrum. The centre frequency of this feature varies with field amplitude and to some extent with the wavelength of the light [fig. 3 (c,d)]. As seen from the $x 10$ expanded view, the characteristic relaxation frequency decreases for longer wavelengths, except for $900 \mathrm{~nm}$ wavelength where the decay is slightly more gradual than for $750 \mathrm{~nm}$ wavelength. The wavelength dependence is more obvious when the field amplitude is larger, for example comparing the minima in the $2.00 \mathrm{mT}$ curves in figs. 4 (d) and 5 (d). The mean intensity of the transmitted light was also recorded but but did not show any clear correlation with the frequency and amplitude of the AC field.

The MNPs respond to an applied field by first aligning with each other to form columns, which may then coalesce ${ }^{9,12}$ on time scales of hundreds of seconds. As the columns thicken, a wavelength dependence of the transmitted light develops that indicates the formation of an optical resonance ${ }^{9}$. This is most relevant for the highest fields used, since the acquisition of the susceptibility data for many different wavelengths may take up to one day. This mechanism may account for the wavelength dependence of both the F1 feature in the optical ACS data and also the second harmonic intensity signal.

The orientation of the easy axis of the chain depends on the strength and the frequency of the applied field ${ }^{18}$. If the field varies slowly then the easy axis has time to reorient parallel to the field. However, the easy axis will exhibit a small oscillatory rotation about a direction orthogonal to the field if the field oscillates with a period shorter than the time needed for full reorientation to occur. The Verdet constant and optical absorption also depend upon the direction of propagation of the light relative to the axis of the chain ${ }^{15}$. These different processes affect the optical ACS data because the bridge detector output is proportional to the intensity of the light, the Verdet constant and the induced magnetization. The data suggests that breakthrough of the intensity variation into the signal from the bridge detector (feature $F 1$ ) is not a simple non-linearity. The amplitude of feature $F 1$ in the optical ACS data decreases with field amplitude in figs. 4 and 5 , but the opposite trend is expected for a non-linear response of the magnetization to the applied field. The measurements made at $550 \mathrm{~nm}$ wavelength, shown in fig. 4, also give insight into the process. Here the $\theta_{F}$ from the MNPs vanishes and the optical ACS signal is dominated by feature $F 1$. The centre frequency of feature $F 1$ follows a similar trend to that of the feature in the second harmonic intensity data, except that the frequency of feature $F 1$ is about three times larger. A similar kind of behavior was reported by Fock et al. ${ }^{20}$ where the peak frequency of the ACS was $\sqrt{3}$ times larger than the second harmonic intensity signal, but with any dispersion of the Brownian relaxation frequency of the MNPs causing this ratio to increase. Therefore the observed factor of about 3 , rather 
than $\sqrt{3}$, suggests a broad dispersion in the hydrodynamic properties of the agglomerates. Alternatively, the enhancement of this factor may also be related to slow agglomeration of the MNPs, since the measurements of the transmitted intensity were performed after the optical ACS measurements A larger aggregate is expected to exhibit a smaller Brownian relaxation frequency that is inversely proportional to its effective hydrodynamic volume ${ }^{19}$.

In conclusion, optical ACS characterisation of magnetite nanoparticles dispersed in a fluid has been performed. The low field optical ACS data depends strongly on the wavelength of the light, while the transmitted intensity is affected by changes in the structural configuration of the fluid. The low frequency feature observed in the intensity signal overlaps with features due to magnetic relaxation within the optical ACS data, and extra care should be taken when fitting the ACS data if the correct magnetic relaxation time is to be extracted. This work has demonstrated the feasibility of using light to probe the ACS of MNPs and paves the way to imaging the ACS of MNPs within biological environments using an optical microscope.

See Supplementary Material for bulk measurements and simulations of the ACS of MNPs dispersed in cyclohexane.

The authors gratefully acknowledge the financial support of the UK Engineering and Physical Sciences Research Council (EPSRC) through research grant EP/P011403/1.

\section{REFERENCES}

${ }^{1} \mathrm{~N}$. Telling, in Nanomaterials for Magnetic and Optical Hyperthermia Applications, edited by R. M. Fratila and J. M. D. L. Fuente (Elsevier, 2019) pp. $173-197$.

${ }^{2}$ D. Soukup, S. Moise, E. Cspedes, J. Dobson, and N. D. Telling, ACS Nano 9, 231 (2015).

${ }^{3}$ D. Cabrera, A. Coene, J. Leliaert, E. J. Arts-Ibez, L. Dupr, N. D. Telling, and F. J. Teran, ACS Nano 12, 2741 (2018).

${ }^{4} \mathrm{R}$. Hergt and S. Dutz, Journal of Magnetism and Magnetic Materials 311 , 187 (2007).

${ }^{5}$ S.-H. Chung, M. Grimsditch, A. Hoffmann, S. D. Bader, J. Xie, S. Peng, and S. Sun, Journal of Magnetism and Magnetic Materials 320, 91 (2008). ${ }^{6}$ M. Ritter, H. Hlsing, and W. P. Wolf, Journal of Applied Physics 55, 2617 (1984)

${ }^{7}$ L. Delaunay, S. Neveu, G. Noyel, and J. Monin, Journal of Magnetism and Magnetic Materials 149, L239 (1995).

${ }^{8}$ R. P. del Real, G. Rosa, and H. Guerrero, Review of Scientific Instruments $\mathbf{7 5}, 2351$ (2004).

${ }^{9}$ J. Jin, D. Song, J. Geng, and D. Jing, Journal of Magnetism and Magnetic Materials 447, 124 (2018)

${ }^{10}$ C. Rablau, P. Vaishnava, C. Sudakar, R. Tackett, G. Lawes, and R. Naik, Physical Review E 78, 051502 (2008)

${ }^{11}$ J. M. Laskar, J. Philip, and B. Raj, Physical Review E 78, 031404 (2008). ${ }^{12}$ N. A. Yusuf, Journal of Physics D: Applied Physics 22, 1916 (1989).

${ }^{13}$ J. M. Laskar, J. Philip, and B. Raj, Physical Review E 80, 041401 (2009).

${ }^{14}$ Y. A. Barnakov, B. Scott, V. Golub, L. Kelly, V. Reddy, and K. Stokes, Journal of Physics and Chemistry of Solids 65, 1005 (2004)

${ }^{15}$ D. A. Smith, Y. A. Barnakov, B. L. Scott, S. A. White, and K. L. Stokes, Journal of Applied Physics 97, 10M504 (2005)

${ }^{16}$ J. Carrey, B. Mehdaoui, and M. Respaud, Journal of Applied Physics 109 , 083921 (2011).

${ }^{17}$ E. Cespedes, J. M. Byrne, N. Farrow, S. Moise, V. S. Coker, M. Bencsik,

J. R. Lloyd, and N. D. Telling, Nanoscale 6, 12958 (2014).

${ }^{18}$ K. Simeonidis, M. P. Morales, M. Marciello, M. Angelakeris, P. de la Presa,

A. Lazaro-Carrillo, A. Tabero, A. Villanueva, O. Chubykalo-Fesenko, and

D. Serantes, Scientific Reports 6, 38382 (2016).

${ }^{19}$ J. Fock, C. Balceris, R. Costo, L. Zeng, F. Ludwig, and M. F. Hansen, Nanoscale 10, 2052 (2018).

${ }^{20}$ J. Fock, C. Jonasson, C. Johansson, and M. F. Hansen, Physical Chemistry Chemical Physics 19, 8802 (2017). 


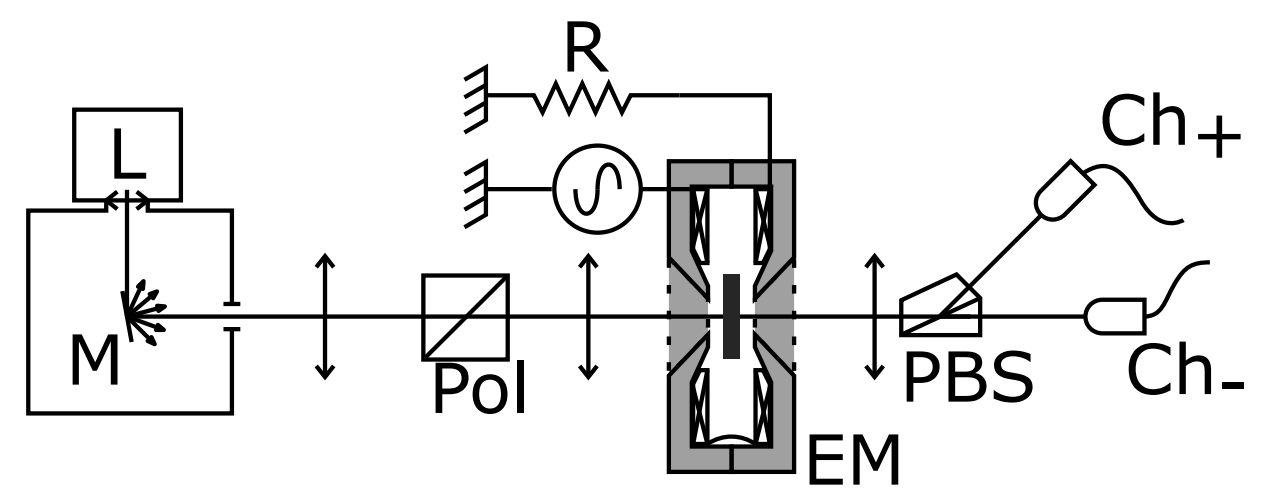

兄旁高 


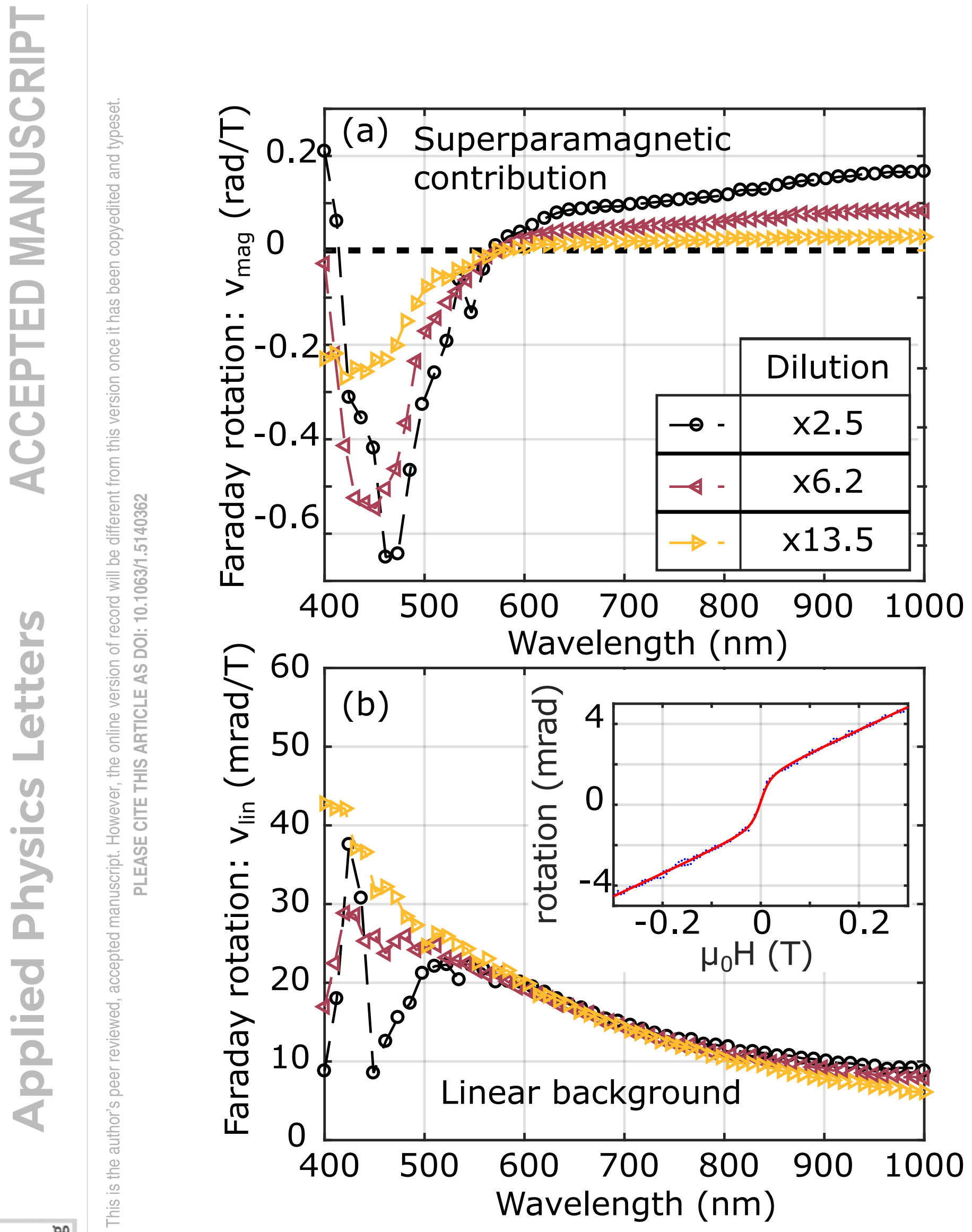




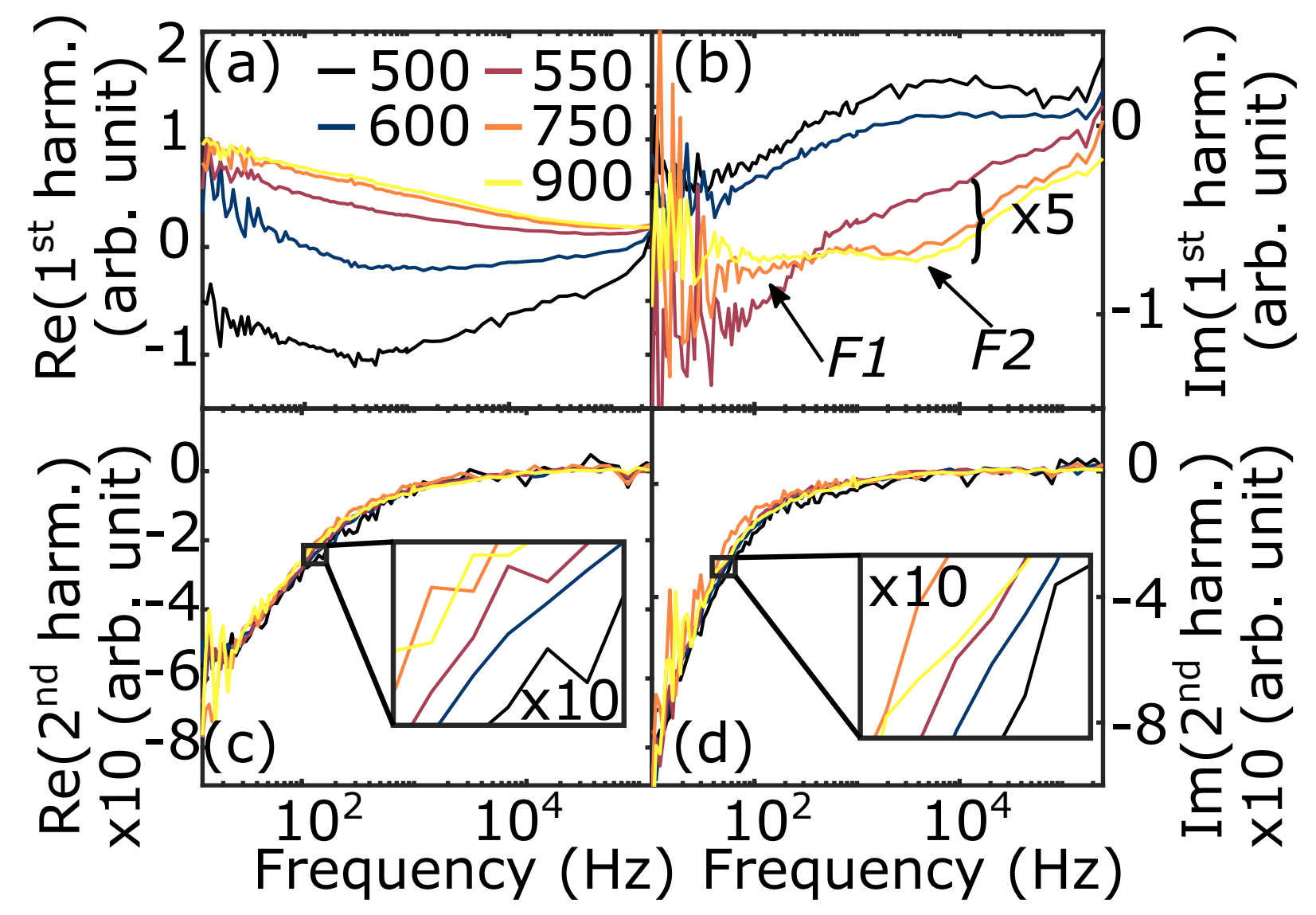




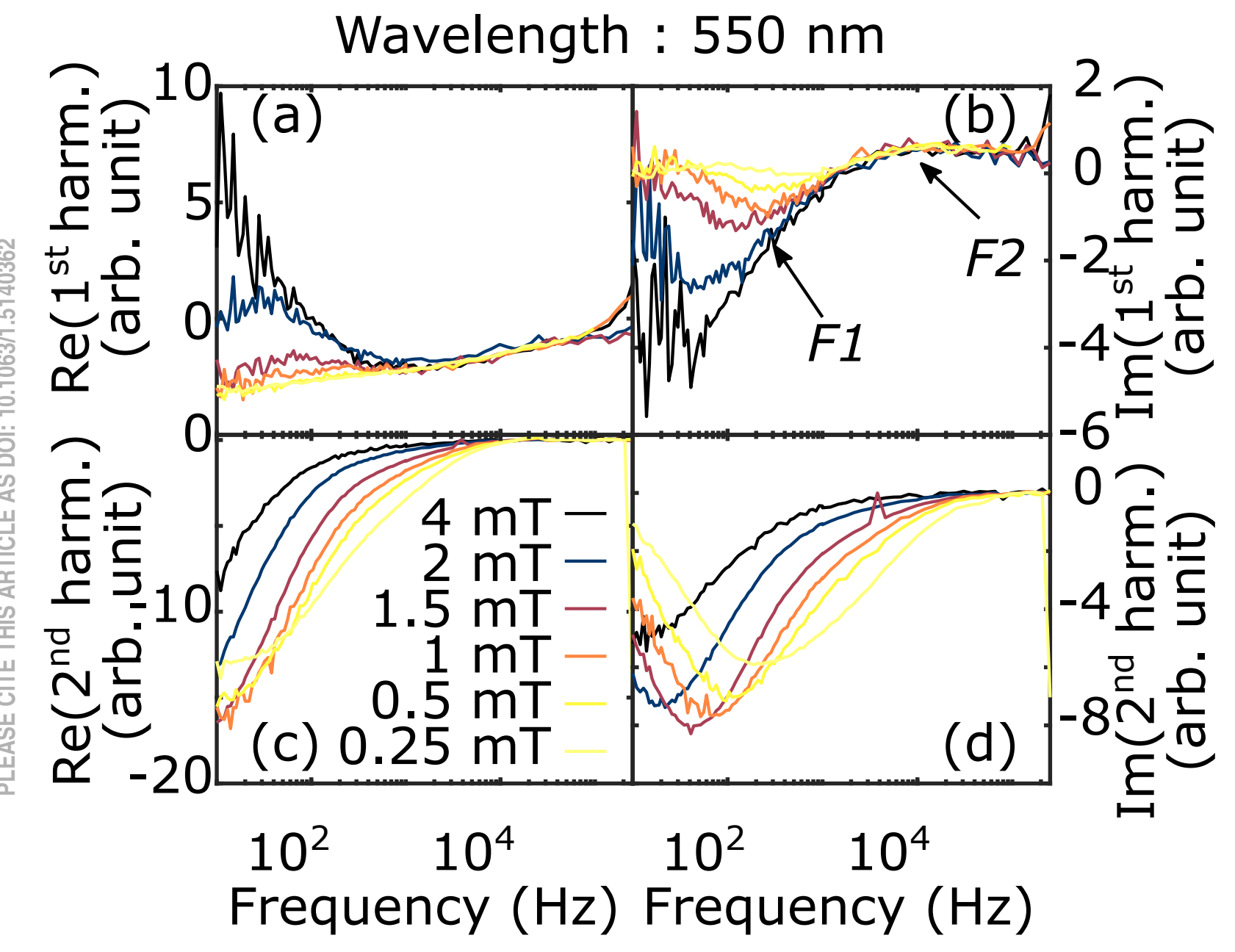




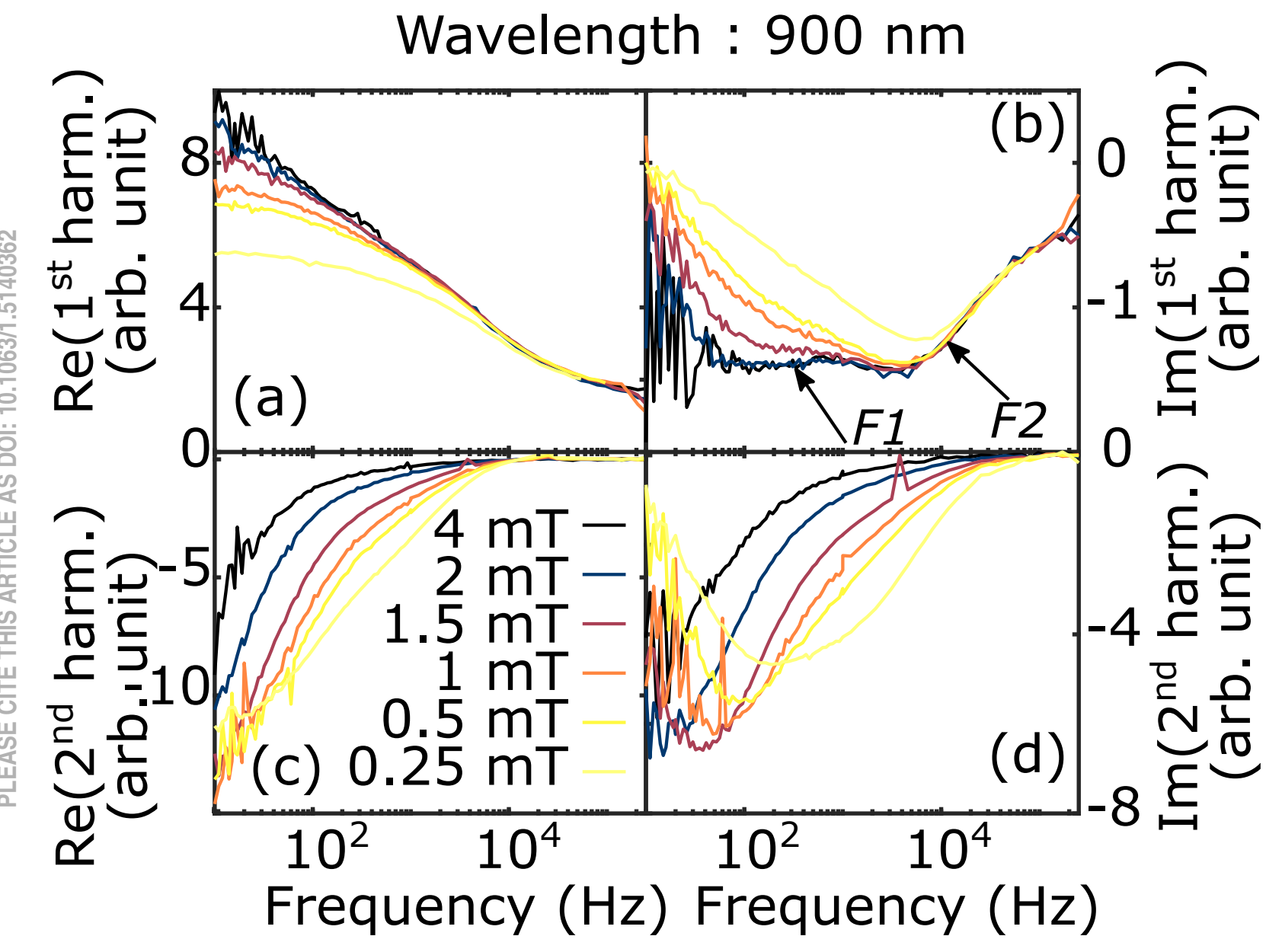

